\title{
Comparación de Software (Plugins) de WORDPRESS para la puesta y marcha de tienda virtual ideal para la micro empresa con modelo de negocio E-Bussines
}

\section{Comparison of WORDPRESS Software (Plugins) for the start-up of an ideal virtual store for the micro-enterprise with an E-Bussines business model}

\author{
SOTO-RODRÍGUEZ, Claudia Aurora*† \& HERNÁNDEZ CERVANTES, Juan \\ Instituto Tecnológico Superior del Occidente del Estado de Hidalgo - Ingeniería en Gestión Empresarial \\ ID $1^{\mathrm{er}}$ Autor: Claudia Aurora, Soto-Rodriguez, ORC ID: 0000-0002-2102-332X, CVU CONACYT ID: 1015285 \\ ID $1^{\text {er }}$ Coautor: Juan, Hernandez-Cervantes / ORC ID: 0000-0002-5332-969X, CVU CONACYT ID: 769796
}

DOI: $10.35429 / J A D .2019 .9 .3 .6 .11$

Recibido 23 de Junio, 2019; Aceptado 17 de Septiembre, 2019

\begin{abstract}
Resumen
Actualmente Wordpress es un sistema de gestión de contenidos(CMS) que permite crear tiendas virtuales (ecommerce) funcionales aplicando los diferentes tipos de software (plugins) que existen en el mercado que permiten aumentar las capacidades y funcionalidades de un modelo de negocio electrónico, el objeto de la presente investigación fue evaluar el alcance, configuración y funcionalidad de los productos compatibles con el CMS, comparando en tiempo real a través de una investigación exploratoria los plugins: ECommerce, Simple Paypal Shopping Cart, Woocommerce, Jigoshop y Ecwid Shopping Cart, en la operación del catálogo de productos de la micro empresa AVIIS avícola S.A. de C.V., el resultado obtenido es la identificación de las características técnicas de cada software para la adaptación de un modelo de negocio electrónico basado en inteligencia de negocio (ebussines) en sus diversas vertientes, los resultados facilitan al empresario de micro empresas la evaluación técnica pertinente de la tecnología a incorporar en su modelo de tienda, para el diseño de estrategias mercadológicas que le permitan mantener o incrementar el posicionamiento de la empresa en el mercado.
\end{abstract}

Plugins, E-Bussines, Wordpress

\begin{abstract}
Wordpress is currently a content management system (CMS) that allows you to create functional virtual stores (e-commerce) by applying the different types of software (plug-ins) that exist in the market that allow you to increase the capabilities and functionalities of an electronic business model, the purpose of the present investigation was to evaluate the scope, configuration and functionality of the products compatible with the CMS, comparing in real time through an exploratory investigation the plug-ins: E-Commerce, Simple Paypal Shopping Cart, Woocommerce, Jigoshop and Ecwid Shopping Cart, in the operation of the product catalog of the micro company AVIIS avícola SA of CV, the result obtained is the identification of the technical characteristics of each software for the adaptation of an electronic business model based on business intelligence (e-bussines) in its various aspects, the results facilitate the evaluation of micro enterprises relevant technology technique to incorporate into your store model, for the design of marketing strategies that allow you to maintain or increase the position of the company in the market.
\end{abstract}

Plugins, E-Bussines, Wordpress

Citación: SOTO-RODRÍGUEZ, Claudia Aurora \& HERNÁNDEZ CERVANTES, Juan. Comparación de Software (Plugins) de WORDPRESS para la puesta y marcha de tienda virtual ideal para la micro empresa con modelo de negocio EBussines. Revista de Arquitectura y Diseño. 2019, 3-9: 6-11

\footnotetext{
*Correspondencia al Autor (Correo Electrónico: caballerogarciasamuel@gmail.com)

$\dagger$ Investigador contribuyendo como primer autor.
} 


\section{Introducción}

Según Mejía \& Sánchez (2015), los factores determinantes de la innovación de los modelos de negocio se basan en la conquista hacia los clientes, dirigida hacia la innovación en la capacitación de la fuerza de ventas, conceptos móviles, experiencias del producto por internet, y productos a domicilio por internet, la creciente demanda de las ventas por internet para la micro empresa se ha vuelto una necesidad preponderante. (Mejía \& Sánchez, 2015)

En México, según reporte de la revista Forbes (2019) el panorama del e-commerce a través del estudio realizado de venta Online en México 2019 por Netrica Netquest, aumenta un $38 \%$ el hábito frecuente en relación al año pasado, ha incrementado la confianza en la compra. (FORBES, 2019) En ese sentido, se destaca que el Sitio o App de las tiendas en línea, se han vuelto las principales fuentes de consulta, generando una mayor penetración de compras en línea por tarjeta de débito, propiciando que los negocios tengan mayor movimiento en sus economías y genera mayor preocupación por lograr mejorar el conocimiento para generar ventas.

El tema central de la presente investigación es identificar el alcance, configuración y funcionalidad necesarios a considerar en la implementación de una tienda en línea, a través de la tecnología que ofrece un sistemas de gestión de contenidos (CMS) en sus siglas en inglés Content Management System, para este caso de estudio se ha considerado Wordpress.

A través de la línea de investigación denominada Empresa, Calidad y Competitividad en la carrera de Ingeniería en Gestión Empresarial se ha desarrollado un prototipo, diseñado a las necesidades de la micro empresa, siendo importante conocer nuevas técnicas en el diseño de las tiendas en línea tomando como base una empresa existente avícola AVIIS S.A de C.V., ubicada en la región occidente del Estado de Hidalgo, así mismo favorecer con un prototipo sencillo de administrar como herramienta de promoción de la empresa incorporando estrategias de mercadotecnia para incrementar las ventas y permitan mejorar el posicionamiento de la empresa en el mercado.
$\mathrm{Al}$ adquirir nuevas técnicas o soluciones para la instalación de tiendas en línea, acorde a las necesidades de la empresa; debe favorecer un nuevo conocimiento especializado que motive a la gestión de la empresa su implementación. Siendo la hipótesis central del estudio "La micro empresa requiere de un proceso de venta en línea más acorde a sus necesidades económicas y técnicas que le permita incrementar su oferta a través del uso de Internet"

Dentro de las características predominantes, se destaca la configuración central del catálogo de productos de la tienda y estrategias de marketing.

El proceso metodológico se basa en una investigación a nivel exploratorio, aplicando como instrumento de recolección de datos bitácoras de uso para evaluar el comportamiento de los elementos básicos de los programas (plugins) que actualmente existen para la instalación y configuración de una tienda en línea, y son compatibles con Wordpress, su evaluación se realizó en tiempo real para registrar el comportamiento de sus componentes. Según Hernández \& Fernández \& Baptista (2010) una investigación exploratoria se realiza cuando el objetivo es examinar un tema o problema de investigación poco estudiado, a un nivel aplicativo, en este caso, existe (Hernández, Fernández, \& Baptista, 2010)

\section{Desarrollo}

\section{Primera Fase}

Al realizar la instalación de Wordpress, en hosting adquirido a través del proveedor externo, se procedió a realizar la búsqueda de los plugins (programas) que actualmente se puede instalar para el diseño del carrito de compras, accediendo a la página oficial del producto en México https://esmx.wordpress.org/, en la sección de Plugins. Según en el blog de EDUAREA (Mayo, 2019), describe que la calidad u disponibilidad de WordPress, en los temas de comercio electrónico hace que sea una plataforma seria para instalar una tienda en línea, ofrece diversos productos para el diseño de tiendas de línea sea flexible a la medida de la empresa. 
A través de la administración se identifica que el espacio a utilizar, verificar las condiciones de la base de datos y es importante revisar el tema o plantilla sea compatible con el plugin a instalar o has seleccionado. (Eduarea Blog, 2019) En ésta primera fase se realizó el proceso de identificación de productos para la instalación de tienda en línea, siendo el caso: ECommerce, Simple Paypal Shopping Cart, Woocommerce, Jigoshop y Ecwid Shopping Cart, identificando las características principales desde la página oficial de descarga del producto, como se muestra en la Tabla 1. Consideraciones básicas de identificación de los Plugins (:

\begin{tabular}{|c|c|c|}
\hline Plugins & Características & Liga de Acceso \\
\hline $\begin{array}{l}\text { Wp E- } \\
\text { commerce }\end{array}$ & $\begin{array}{l}\text { Ideal para pequeños negocios Escala } \\
\text { a un catálogo de productos de amplia } \\
\text { gama. } \\
\text { Limita proceso de pago integral } \\
\text { Salida segura con SSL }\end{array}$ & $\begin{array}{l}\text { https://wordpress. } \\
\text { org/plugins/wp-e- } \\
\text { commerce/ }\end{array}$ \\
\hline $\begin{array}{l}\text { Simple Paypal } \\
\text { Shopping Cart }\end{array}$ & $\begin{array}{l}\text { Fácil añade botón de carrito } \\
\text { dinámica, con shortcode. } \\
\text { Cantidad mínima de elementos de } \\
\text { configuración } \\
\text { Cualquier tipo de productos tangibles } \\
\text { de su sitio. } \\
\text { Mostrar una caja de exposición de } \\
\text { productos con un formato agradable. } \\
\text { Zona de pruebas de PayPal } \\
\text { Configurar cupones de descuento. } \\
\text { Compatible con la instalación de } \\
\text { WordPress multisitio. } \\
\text { Puede ser traducido en varios } \\
\text { idiomas. }\end{array}$ & $\begin{array}{l}\text { https://wordpress. } \\
\text { org/plugins/wordp } \\
\text { ress-simple- } \\
\text { paypal-shopping- } \\
\text { cart/ }\end{array}$ \\
\hline $\begin{array}{l}\text { Woocommerc } \\
\text { e }\end{array}$ & $\begin{array}{l}\text { Acceso remoto MySQL a la base de } \\
\text { datos osCommerce. } \\
\text { Todos los productos y categorías se } \\
\text { importarán de una vez, no } \\
\text { escalonada. } \\
\text { Si hay suficiente demanda, se agrega } \\
\text { sistema para evitar tiempos de } \\
\text { espera. }\end{array}$ & $\begin{array}{l}\text { https://wordpress. } \\
\text { org/plugins/woo- } \\
\text { oscommerce- } \\
\text { sync/ }\end{array}$ \\
\hline Jigoshop & 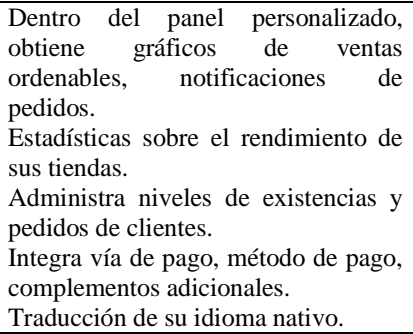 & $\begin{array}{l}\text { https://wordpress. } \\
\text { org/plugins/jigosh } \\
\text { op-ecommerce/ }\end{array}$ \\
\hline $\begin{array}{l}\text { Ecwid } \\
\text { Shopping Cart }\end{array}$ & $\begin{array}{l}\text { Potente, fácil de usar y amigable. } \\
\text { Principales operadores, incluidos } \\
\text { USPS, UPS, FedEx, Canada Post, } \\
\text { Australia Post y otros para calcular } \\
\text { automáticamente las tarifas de envío. } \\
\text { Ecwid Shopping Cart ofrece } \\
\text { aplicaciones móviles gratuitas para } \\
\text { iOS y Android para administrar su } \\
\text { tienda y vender sobre la marcha. } \\
\text { Los datos de su tienda en línea } \\
\text { alojados en servidores seguros. } \\
\text { Puede incluir su tienda de comercio } \\
\text { electrónico en múltiples sitios web, } \\
\text { blogs, sitios sociales y mercados de } \\
\text { comercio electrónico. }\end{array}$ & $\begin{array}{l}\text { https://wordpress. } \\
\text { org/plugins/ecwid } \\
\text {-shopping-cart/ }\end{array}$ \\
\hline
\end{tabular}

Tabla 1 Consideraciones básicas de identificación de los Plugins

Fuente: Elaboración propia consulta en (Wordpress.org, 2019)

\section{Segunda Fase}

\section{Instalación simultánea de los productos en Worpress}

Para el estudio fue importante la instalación simultánea de los productos con la finalidad de analizar de acuerdo a la versión compatible de Wordpress 5.2.2, la pertinencia de cada producto en la configuración inicial del carrito de compras, validando principalmente el alcance y configuración de cada configuración:

En la Tabla 2. Alcance y aspectos de configuración, se describe algunas características de cada producto para la creación de una Tienda en línea, en la instalación de cada producto se realizó su descarga en página oficial de WordPress en opción de Plugin, se descomprime cada carpeta para ser alojados en carpeta de wp-content, para posteriormente ser activado en el panel del administrador:

\begin{tabular}{|c|c|c|}
\hline Producto & Alcance & $\begin{array}{l}\text { Aspectos de } \\
\text { configuración }\end{array}$ \\
\hline $\begin{array}{ll}\text { Wp } & \text { E- } \\
\text { commerce }\end{array}$ & $\begin{array}{l}\text { Limitado } \\
\text { Errores de instalación, } \\
\text { versión no compatible con } \\
\text { wordpress 5.2.2. }\end{array}$ & $\begin{array}{l}\text { Se requiere encontrar } \\
\text { versión compatible }\end{array}$ \\
\hline $\begin{array}{l}\text { Simple Paypal } \\
\text { Shopping Cart }\end{array}$ & $\begin{array}{l}\text { Sencillo } \\
\text { Integra sólo proceso de } \\
\text { catálogo de productos } \\
\text { Cuenta } \\
\text { No proceso de envío y } \\
\text { facturación }\end{array}$ & $\begin{array}{l}\text { Ajuste de lugar de } \\
\text { negocio y tipo de } \\
\text { moneda } \\
\text { Conexión con Paypal } \\
\text { Shortcot sencillo } \\
\text { Configura cupones } \\
\text { Exporta datos } \\
\end{array}$ \\
\hline $\begin{array}{l}\text { Woocommerce } \\
3.5 .1\end{array}$ & $\begin{array}{l}\text { Sólo conexión con } \\
\text { internet } \\
\text { Ofrece varias opciones de } \\
\text { pago. } \\
\text { Asistente de configuración } \\
\text { sencillo en } 6 \text { pasos } \\
\text { Pantalla amigable en } \\
\text { configuración de catálogo } \\
\text { de productos. } \\
\text { Creación inmediata de } \\
\text { productos }\end{array}$ & $\begin{array}{l}\text { A través del proceso de } \\
\text { asistente } \\
\text { Considerar imágenes } \\
\text { de productos, } \\
\text { seguimiento de pedido } \\
\text { Conexión con Jetpack } \\
\text { Conexión Facebook } \\
\text { Para promoción de } \\
\text { página }\end{array}$ \\
\hline Jigoshop & $\begin{array}{l}\text { Conexión con internet } \\
4 \text { pasos de configuración } \\
\text { Asistente de configuración } \\
\text { sencillo } \\
\text { Genera } 7 \text { páginas de } \\
\text { seguimiento del pedido } \\
\text { Varias opciones de pago } \\
\text { Sin costo }\end{array}$ & $\begin{array}{l}\text { Datos generales de la } \\
\text { empresa, varias } \\
\text { opciones de pago, } \\
\text { menú no sencillo en } \\
\text { cada fase } \\
\text { Contenido, daswboard, } \\
\text { reportes } \\
\begin{array}{l}\text { Definiciones } \\
\text { cupones }\end{array}\end{array}$ \\
\hline $\begin{array}{l}\text { Ecwid Shopping } \\
\text { Cart }\end{array}$ & $\begin{array}{l}5 \text { pasos de creación } \\
\text { Creación de cuenta } \\
\text { Conexión con internet } \\
\text { Define varios canales de } \\
\text { entrega de pedido } \\
\text { Asistente de configuración } \\
\text { muy sencillo } \\
\text { Módulo de administración } \\
\text { muy sencillo }\end{array}$ & $\begin{array}{l}\text { Integra un primer } \\
\text { producto de muestra } \\
\text { Permite borrador de } \\
\text { tienda } \\
\text { Verifica la configuración } \\
\text { geográfica para } \\
\text { segmento de mercado } \\
\text { Integra un panel integral } \\
\text { la gestión de la tienda }\end{array}$ \\
\hline
\end{tabular}

Tabla 2 Alcance y aspectos de configuración de la Tienda en Línea

Fuente: Elaboración propia, acceso a servidor local 


\section{Tercera Fase}

Al comparar las diversas modalidades que se ofrecen en cada programa (plugin), se realiza la integración de catálogo de productos, en función de la definición de los parámetros de condiciones para su venta, en la Tabla 3. Parámetros básicos de integración de catálogo de productos de Ecwid Shopping Cart:

\begin{tabular}{|l|l|}
\hline \multicolumn{1}{|c|}{ Aspectos } & \multicolumn{1}{|c|}{ Características } \\
\hline Lote & $\begin{array}{l}\text { Envío de imágenes por lotes, archivo } \\
\text { CSV UTF-8 separado por punto y coma } \\
\text { o archivo Xcart tabulador, integrando } \\
\text { columnas básicas nombre, REF, } \\
\text { descripción, categorías, URL, precio, } \\
\text { cantidad }\end{array}$ \\
\hline Individual & $\begin{array}{l}\text { Creación por producto, ingresando } \\
\text { nombre, REF, peso, descripción, } \\
\text { atributos, opciones y SEO }\end{array}$ \\
\hline Categorías & $\begin{array}{l}\text { Es importante considerar categorías o } \\
\text { clasificación del producto para una } \\
\text { búsqueda ordenada y mejorar vista al } \\
\text { cliente. }\end{array}$ \\
\hline Marketing & $\begin{array}{l}\text { Opción de integrar campaña de } \\
\text { promoción por Google y Facebook } \\
\text { Promociones, cupones, boletines } \\
\text { electrónicos, analizar el rendimiento } \\
\text { con Google Analytics para medición de } \\
\text { indicadores }\end{array}$ \\
\hline Canales de & $\begin{array}{l}\text { Vender en Facebook, Instagram, } \\
\text { Messenger, Móvil, Starter Site, Google } \\
\text { Shooping, Vend POS, eBay, Amazon }\end{array}$ \\
Ventas & $\begin{array}{l}\text { Diseño de página a empresa mediana } \\
\text { Pago } \\
\text { Envío y recogida del producto }\end{array}$ \\
\hline Configuración \\
\hline
\end{tabular}

Tabla 3 Parámetros básicos de integración de catálogo de productos de Ecwid Shopping Cart

Fuente: Elaboración propia, acceso a servidor local

\section{Resultados}

La comparación de productos a nivel exploratoria permitió identificar el programa (plugin) más idóneo, por su alcance, configuración, funcionalidad y sencillez, las pruebas se realizaron en servidor local, que permitió enviarlo a hosting o alojamiento de página de proveedor externo, actualmente funciona a nivel de pruebas estamos en proceso con la empresa AVIIS S.A. de C.V. para la validez del catálogo de productos, promociones, campañas de publicidad y diseño de página. Se cuenta con prototipo ajustado a las necesidades de la empresa pero también estándar que puede servir a cualquier micro empresa de la región occidente del estado de Hidalgo.
A continuación, se realiza demostración de imágenes de cada una de las fases realizadas en la implementación del prototipo:

En la figura 1. Diseño de catálogo de los productos, a través del módulo correspondiente a la aplicación se fueron dando de alta cada uno de los productos, con descripción precios y stock, así como la definición de categorías.

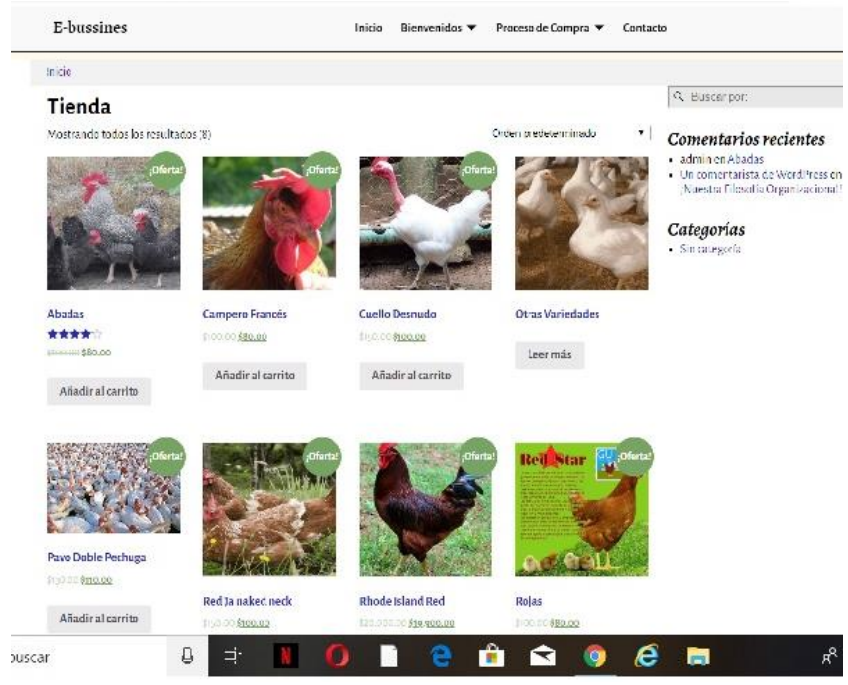

Figura 1 Diseño del Catálogo de los Productos

Fuente: Consulta interactiva de la plataforma del servidor local. http://www.investitsoehige.com.mx/ebussines/tienda/

En la Figura 2. Diseño de Markting, en este módulo se dio como entrada principal mostrar la filosofía organizacional de la empresa, y también el alta de promociones de cada una de las opciones de los productos

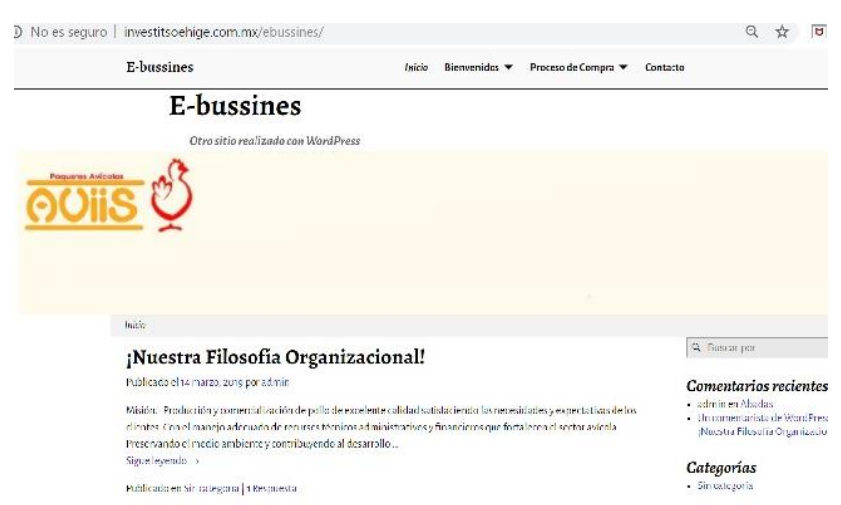

Figura 2 Diseño de Marketing

Fuente: Consulta interactiva de la plataforma del servidor local 


\section{Diseño de Canales de Venta}

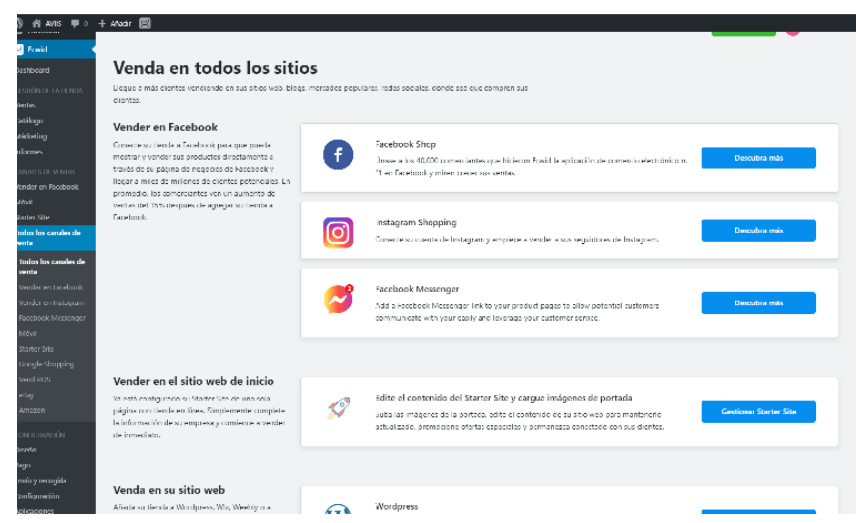

Figura 3 Diseño de Canales de Venta

Fuente: Consulta interactiva de la plataforma del servidor local.http://localhost/aviis/wpadmin/admin.php?page=ec-store-admin-sales-channel

\section{Diseño de Configuración}
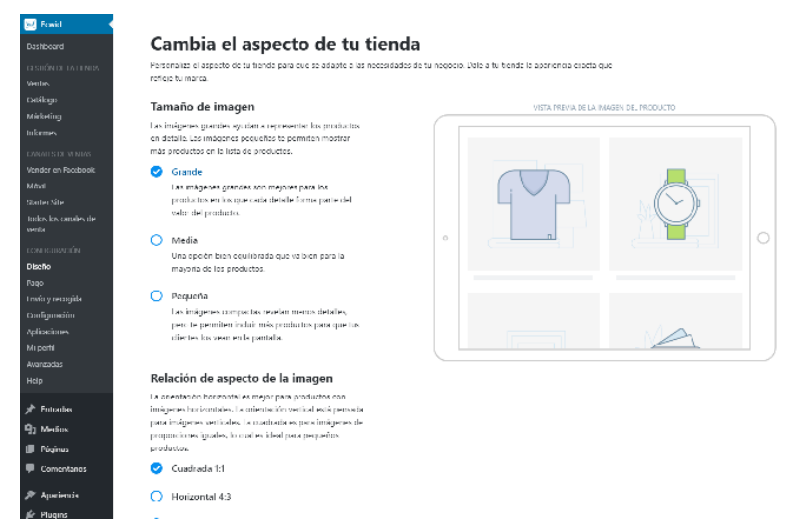

Figura 4 Diseño de Configuración

Fuente: Consulta interactiva de la plataforma del servidor local

Al integrar la propuesta del prototipo actualmente se continúa trabajando con la empresa para definir el estudio del diseño de la cadena de suministro que implique el proceso completo de la entrega de los productos a los clientes.

\section{Agradecimiento}

Agradecemos al Tecnológico Nacional de México por el financiamiento otorgado al proyecto denominado "Market E-Bussines Inteligencia de negocio para Mypymes" bajo convocatoria 2019, permitirá brindar a las micro empresas de la Región Occidente del Estado de Hidalgo, México, la oportunidad de adquirir nuevos conocimientos en la administración de su negocio, permitiendo que la toma de decisiones sea de una manera más inteligente y asertiva.

\section{Conclusiones}

La idea central del estudio, era probar el nivel de eficiencia y funcionalidad que ofrece WordPress, con los diversos programas (plugins) que ofrece para la instalación de la Tienda en Línea, logrando obtener un prototipo ágil, sencillo y funcional del carrito de compras, de los resultados obtenidos está permitiendo realizar un instructivo o manual para brindarlo a las empresas de la región que les faciliten de manera sencilla la instalación propia de su tienda en línea.

Otro aspecto importante identificado que el producto Ecwid Shopping Cart y Jigoshop, ofrecen un proceso de monitoreo de indicadores para el seguimiento del pedido y estatus actual en tiempo real.

Actualmente la inteligencia de negocios se vuelve imprescindible para la toma de decisiones, los beneficios que cada producto son importante, dependerá de la necesidad de la empresa y la forma en que desea iniciar con las ventas en línea, el nivel de pago y otros requisitos desea ofrecer.

La integración de diversas tecnologías del carrito de compras fue importante para garantizar un proceso completo de compraventa, estabilidad y funcionalidad, La visión empresarial principalmente de las MyPymes, deben estar acordes a los avances tecnológicos, sólo basta romper con paradigmas tradicionales que fortalezcan su reconocimiento en el mercado.

\section{Referencias}

Eduarea Blog. (2019). 16 características más importantes de una buena empresa de alojamiento web. Obtenido de https://eduarea.wordpress.com/2019/03/03/16caracteristicas-mas-importantes-de-una-buenaempresa-de-alojamiento-web/

FORBES. (5 de Marzo de 2019). El panorama de e-commerce en México en 2019. FORBES México, pág. 1. Obtenido de https://www.forbes.com.mx/el-panorama-de-ecommerce-en-mexico-en-2019/

Hernández, S. J., Fernández, C. C., \& Baptista, L. M. (2010). Metodología de la Investigación. México: Mc Graw Hill. 
Mejía, J., \& Sánchez, J. (2015). Factores determinantes de la innovación del modelo de negocios en la. Revista del Centro de Investigación RECEIN, Vol. 11(No. 42), 105128.

Obtenido de http://www.redalyc.org/pdf/342/34235376004.p df

Wordpress.org. (Septiembre de 2019). Wordpress. Obtenido de https://wordpress.org/plugins 